\title{
Are Trading Rules Profitable in Exchange-Traded Funds?
}

\author{
Terence Tai-Leung Chong ${ }^{1}$, Elton Hei-Tung $\mathrm{Li}^{2}$, Kenneth Tak-Kan Kong ${ }^{2}$ \\ ${ }^{1}$ Department of Economics and Hong Kong Institute of Asia-Pacific Studies, \\ The Chinese University of Hong Kong, Hong Kong, China \\ ${ }^{2}$ Department of Economics, The Chinese University of Hong Kong, Hong Kong, China \\ E-mail: chong2064@cuhk.edu.hk \\ Received March 1, 2011; revised April 28, 2011; accepted May 4, 2011
}

\begin{abstract}
The Exchange-traded fund (ETF) is a burgeoning financial vehicle. Despite its growing importance, there has been a lack of empirical studies on the profitability of technical trading rules in the ETF market. This paper assesses the profitability of the On-Balance Volume indicator (OBV) on ETF trading. It is found that the trading rules associated with the OBV are able to generate handsome returns in the ETF market. This is in contrast to the conventional wisdom that funds should be bought and held.
\end{abstract}

Keywords: On-Balance Volume, Exchange-Traded Funds, Moving Average

\section{Introduction}

A wide variety of nascent financial vehicles have been developed in recent years. In particular, the Exchangetraded fund (ETF), among other financial innovations, has become a burgeoning financial vehicle and gained the attention of the populace. ETFs have a relatively short history. The world's first ETF was launched in 1993 , with a total asset value of US $\$ 484$ million. By the end of 2010, there are over 1000 ETFs traded on US exchanges, with a total asset value of more than US $\$ 790$ billion, reflecting a growing demand for this new financial vehicle. ${ }^{1}$ Compared to mutual funds, ETFs offer have a variety of advantages, such as a greater flexibility, lower fee, increased tax efficiency and greater transparency [1]. ${ }^{2}$ Traditional performance metrics, such as Price/Earnings ratio and cash flow, cannot be applied to trade ETFs [2]. This paper examines a topical research area. Following Tsang and Chong [3], we evaluate the performance of the On-Balance Volume (OBV) indicator in the ETF market. The $O B V$ is a simple indicator defined as:

$$
O B V_{t}=\left\{\begin{array}{l}
O B V_{t-1}+V_{t}, C_{t}>C_{t-1} \\
O B V_{t-1}-V_{t}, C_{t}<C_{t-1} \\
O B V_{t-1}, C_{t}=C_{t-1}
\end{array}\right.
$$

where $C_{t}$ and $V_{t}$ are the closing price and trading volume

\footnotetext{
${ }^{1}$ The exact number of ETFs depends on the definition employed, as there are many other ETF-like products listed in different exchanges [1].

${ }^{2}$ For recent studies on ETFs, see [4] and [5].
}

respectively at time $t$. We let $O B V_{0}$ to be zero. A rising price together with a rising $O B V$ indicates that the volume is heavier on up days, confirming the uptrend of the market. If prices are moving higher while the volume is falling, it suggests a trend reversal.

\section{Data and Methodology}

Our sample includes 30 US listed ETFs ${ }^{3}$ from six different categories, namely, Bear Market, Latin America, Telecommunications, Consumers Staples, Bond and Government Treasury Bills. ${ }^{4}$ Following Tsang and Chong [3], we employ the trading rules associated with the $O B V$ indicator. ${ }^{5}$ Five trading rules are studied. The first four rules are based on the crossing of $O B V$ and its n-day moving average, which is defined as:

$$
O B V M A_{t}^{n}=\frac{1}{n} \sum_{i=1}^{n} O B V_{i}
$$

A trading signal is produced when $O B V$ crosses the corresponding moving average. The fifth rule is based on the crossing of two $O B V$ moving averages. The five $O B V$ rules are defined as follows:

${ }^{3}$ According to the definition of Morning Star, ETFs that mainly hold US Treasury Bills are categorized as Short Government, Intermediate Government and Long Government. In our analysis, we define US Treasury Bills category as any ETFs falling into these three categories.

${ }^{4}$ These classifications, except for US Treasury Bills, are set by the ETFs' corresponding issuers.

${ }^{5}$ We derive the OBV indicator using the trading volume of the ETFs, instead of the trading volume of the underlying assets. 
Rule 1: 10-day OBV

Buy at day $t: O B V_{t-1}<O B V M A_{t-1}^{10}$ and $O B V_{t}>O B V M A_{t}^{10}$

Sell at day $t: O B V_{t-1}>O B V M A_{t-1}^{10}$ and $O B V_{t}<O B V M A_{t}^{10}$

Rule 2: 20-day $O B V$

Buy at day $t: O B V_{t-1}<O B V M A_{t-1}^{20}$ and $O B V_{t}>O B V M A_{t}^{20}$

Sell at day $t: O B V_{t-1}>O B V M A_{t-1}^{20}$ and $O B V_{t}<O B V M A_{t}^{20}$

Rule 3: 50-day OBV

Buy at day $t: O B V_{t-1}<O B V M A_{t-1}^{50}$ and $O B V_{t}>O B V M A_{t}^{50}$

Sell at day $t: O B V_{t-1}>O B V M A_{t-1}^{50}$ and $O B V_{t}<O B V M A_{t}^{50}$

Rule 4: 100-day OBV

Buy at day $t: O B V_{t-1}<O B V M A_{t-1}^{100}$ and $O B V_{t}>O B V M A_{t}^{100}$

Sell at day $t: O B V_{t-1}>O B V M A_{t-1}^{100}$ and $O B V_{t}<O B V M A_{t}^{100}$

The fifth trading rule, which is based on the crossing of 10-day OBVMA and 20-day OBVMA, is defined as follows:

Rule 5: OBVMA10 $\times 20$

Buy at day $t: O B V M A_{t-1}^{10}<O B V M A_{t-1}^{20}$ and $O B V M A_{t}^{10}>O B V M A_{t}^{20}$

Sell at day $t: O B V M A_{t-1}^{10}>O B V M A_{t-1}^{20}$ and

\section{$O B V M A_{t}^{10}<O B V M A_{t}^{20}$}

For the first four rules, a long position will be taken if $O B V$ rises above the corresponding $O B V M A$, and liquidated when $O B V$ falls below the corresponding OBVMA. The choices of the four window length $n=10,20,50$ and 100 are common (Brock et al. [6], Tsang and Chong [3]). If a smaller value of $n$ is used, there will be many noisy signals. For long-term investment, one may use a larger value of $n$ to reduce the number of transactions.

We prohibit short-selling and consecutive buying/ selling in our calculation of returns. ${ }^{6}$ Transaction cost is assumed to be negligible. The performance of the five trading rules is evaluated in terms of the annualized rate of return. Since there are about 250 trading days each year, the annualized rate of return can be defined as follows:

$$
R_{A}=\left[\left(1+r_{1}\right)\left(1+r_{2}\right)\left(1+r_{3}\right) \ldots\left(1+r_{\mathrm{m}}\right)\right]^{250 / T}-1
$$

where

$$
1+r_{i}=S(i) / B(i),
$$

$S(i)$ and $B(i)$ are respectively the selling and buying prices of the $i^{\text {th }}$ transaction;

$m$ is the number of transactions in the sample;

$T$ is the number of trading days in the sample. ${ }^{7}$

To assess the impact of the 2008 Financial Tsunami on the profitability of our trading rules, we further split the whole sample into Pre-Tsunami and Post-Tsunami subsamples. The day when Lehman Brothers Holdings Inc. was delisted from the New York Stock Exchange, 17 ${ }^{\text {th }}$ September 2008, was set as the watershed. This is the major event that marks the onset of the Financial Tsunami. The daily closing prices and volume of each ETF are gleaned from DataStream. The details are reported in Table 1.

Table 1. ETF name list and time period.

\begin{tabular}{|c|c|c|c|c|}
\hline Ticker & ETF Name & Overall & Pre-Tsunami & Post-Tsunami \\
\hline SIJ & ProShares UltraShort Industrials & \multirow{8}{*}{$19 / 3 / 07-17 / 3 / 10$} & \multirow{8}{*}{$19 / 3 / 07-17 / 9 / 08$} & \multirow{8}{*}{$18 / 9 / 08-17 / 3 / 10$} \\
\hline $\mathrm{SH}$ & ProShares Short S\&P500 & & & \\
\hline DOG & ProShares Short Dow30 & & & \\
\hline UDN & $\begin{array}{c}\text { PowerShares DB US Dollar Index } \\
\text { Bearish Fund }\end{array}$ & & & \\
\hline SRS & ProShares UltraShort Real Estate & & & \\
\hline SMN & ProShares UltraShort Basic Materials & & & \\
\hline SKF & ProShares UltraShort Financials & & & \\
\hline DUG & Proshares UltraShort Oil \& Gas & & & \\
\hline
\end{tabular}

Bear Market Category

${ }^{6}$ Short selling means the short selling of the ETF itself. ETFs in the Bear Market Category sometimes adopt a strategy of short-selling its underlying asset.
${ }^{7}$ Suppose we start with one dollar of investment, then $\left[\left(1+r_{1}\right)\left(1+r_{2}\right)(1+\right.$ $\left.\left.r_{3}\right) \ldots\left(1+r_{m}\right)\right]$ will be the total return after all the $\mathrm{m}$ transactions during period T. After taking the power of $250 / \mathrm{T}$, and less the initial one dollar, we obtain the annualized rate of return. 
Latin America Category

\begin{tabular}{|c|c|c|c|c|}
\hline Ticker & ETF Name & Overall & Pre-Tsunami & Post-Tsunami \\
\hline EWZ & iShares MSCI Brazil Index Fund & \multirow[b]{2}{*}{$19 / 3 / 07-17 / 3 / 10$} & \multirow[b]{2}{*}{$19 / 3 / 07-17 / 9 / 08$} & \multirow[b]{2}{*}{$18 / 9 / 08-17 / 3 / 10$} \\
\hline ILF & $\begin{array}{c}\text { iShares S\&P Latin America } 40 \text { Index } \\
\text { Fund }\end{array}$ & & & \\
\hline \multicolumn{5}{|c|}{ Telecommunications Category } \\
\hline Ticker & ETF Name & Overall & Pre-Tsunami & Post-Tsunami \\
\hline IXP & $\begin{array}{l}\text { iShares S\&P Global Telecom- } \\
\text { munications Sector Index Fund }\end{array}$ & & & \\
\hline VOX & $\begin{array}{l}\text { Vanguard Telecommunication } \\
\text { Services ETF }\end{array}$ & $19 / 3 / 07-17 / 3 / 10$ & $19 / 3 / 07-17 / 9 / 08$ & $18 / 9 / 08-17 / 3 / 10$ \\
\hline PBS & $\begin{array}{c}\text { PowerShares Dynamic Media } \\
\text { Portfolio }\end{array}$ & & & \\
\hline
\end{tabular}

\section{Consumer Staples Category}

\begin{tabular}{|c|c|c|c|c|}
\hline Ticker & ETF Name & Overall & Pre-Tsunami & Post-Tsunami \\
\hline XLP & $\begin{array}{l}\text { Consumer Staples Select Sector } \\
\text { SPDR }\end{array}$ & 19/3/07-17/3/10 & 19/3/07-17/9/08 & 18/9/08-17/3/10 \\
\hline VDC & Vanguard Consumer Staples ETF & & & \\
\hline
\end{tabular}

\section{Bond Category}

\begin{tabular}{lllll}
\hline Ticker & ETF Name & Overall & Pre-Tsunami & Post-Tsunami \\
\hline HYG & iShares iBoxx \$ High Yield Corporate Bond & $11 / 4 / 07-17 / 3 / 10$ & $11 / 4 / 07-17 / 9 / 08$ \\
& Fund & & \\
GBF & iShares Barclays Government/Credit Bond & $13 / 3 / 07-17 / 3 / 10$ & $13 / 3 / 07-17 / 9 / 08$ \\
& Fund & & \\
BSV & Vanguard Short-Term Bond ETF & $10 / 4 / 07-17 / 3 / 10$ & $10 / 4 / 07-17 / 9 / 08$ \\
BLV & Vanguard Long-Term Bond ETF & $10 / 4 / 07-17 / 3 / 10$ & $10 / 4 / 07-17 / 9 / 08$ \\
BIV & Vanguard Intermediate-Term Bond ETF & $10 / 4 / 07-17 / 3 / 10$ & $10 / 4 / 07-17 / 9 / 08$ & $18 / 9 / 08-17 / 3 / 10$ \\
CSJ & iShares Barclays 1-3 Year Credit Bond Fund & $19 / 3 / 07-17 / 3 / 10$ & $19 / 3 / 07-17 / 9 / 08$ & $19 / 3 / 07-17 / 9 / 08$ \\
GVI & iShares Barclays Intermediate Govern- & $19 / 3 / 07-17 / 3 / 10$ & & \\
& ment/Credit Bond Fund & & $19 / 3 / 07-17 / 9 / 08$ \\
LQD & iShares iBoxx \$ Investment Grade Corporate & $19 / 3 / 07-17 / 3 / 10$ & & \\
& Bond Fund & $19 / 3 / 07-17 / 3 / 10$ & $19 / 3 / 07-17 / 9 / 08$ \\
TIP & iShares Barclays TIPS Bond Fund & $16 / 3 / 07-17 / 3 / 10$ & $16 / 3 / 07-17 / 9 / 08$ \\
\hline MBB & iShares Barclays MBS Bond Fund & & \\
\hline
\end{tabular}

\section{U.S. Treasury Bill Category}

\begin{tabular}{lllll}
\hline Ticker & ETF Name & Overall & Pre-Tsunami & Post-Tsunami \\
\hline IEI & iShares Barclays 3-7 Year Treasury Bond & & \\
& Fund & & & \\
SHV & iShares Barclays Short Treasury Bond & & \\
& Fund & & & \\
SHY & iShares Barclays 1-3 Year Treasury Bond & $19 / 3 / 07-17 / 3 / 10$ & $19 / 3 / 07-17 / 9 / 08$ & $18 / 08-17 / 3 / 10$ \\
& Fund & & \\
TLH & iShares Barclays 10-20 Year Treasury Bond & & \\
& Fund & & & \\
TLT & iShares Barclays 20+ Year Treasury Bond & & \\
& Fund &
\end{tabular}

Table 2. a. Annual Rate of Return; b. Annual Rate of Return; c. Annual Rate of Return; d. Annual Rate of Return; e. Annual Rate of Return; f. Annual Rate of Return.

a. Bear Market Category

\begin{tabular}{|c|c|c|c|c|c|c|c|c|c|c|c|c|c|c|c|c|c|c|}
\hline \multirow[b]{2}{*}{ Ticker } & \multirow[b]{2}{*}{ Volatility } & \multicolumn{6}{|c|}{ Overall $(19 / 03 / 07-17 / 03 / 10)$} & \multicolumn{6}{|c|}{ Pre-Tsunami (19/03/07-17/09/08) } & \multicolumn{5}{|c|}{ Post- Tsunami $(18 / 09 / 08-17 / 03 / 10)$} \\
\hline & & Buy-hold & OBV10 & OBV20 & OBV50 & OBV100 & OBVMA $10 \times 20$ & Buy-hold & OBV10 & OBV20 & 0 OBV50 & OBV100 & OBVMA10 $\times 20$ & Buy-hold & OBV10 OBV20 & 0 OBV50 & OBV100 & OBVMA10 $\times 20$ \\
\hline SIJ & $61 \%$ & -33.97 & -22.03 & -32.89 & -37.07 & -0.99 & -35.33 & 7.55 & -3.58 & 10.89 & 11.32 & 10.33 & -2.24 & -56.18 & $-49.36-56.01$ & -67.62 & -26.52 & -54.51 \\
\hline SH & $27 \%$ & -7.96 & -14.42 & 0.16 & -9.28 & -8.25 & 2.25 & 10.89 & -9.34 & 4.12 & -2.05 & 0.60 & 17.73 & -22.01 & $-19.26-10.99$ & -17.92 & -8.29 & -3.40 \\
\hline
\end{tabular}




\begin{tabular}{|c|c|c|c|c|c|c|c|c|c|c|c|c|c|c|c|c|c|c|c|}
\hline DOG & $24 \%$ & -8.03 & -5.88 & 0.31 & -7.71 & -5.01 & 4.31 & 7.25 & -7.65 & 2.29 & 3.92 & 3.73 & 11.44 & -19.35 & -4.06 & -6.27 & -15.38 & -2.79 & -2.44 \\
\hline UDN & $10 \%$ & 2.24 & 3.91 & 2.22 & 1.96 & 2.93 & 4.19 & 6.03 & 8.44 & 1.54 & -0.16 & 5.15 & 2.52 & -1.26 & 0.80 & 3.50 & 4.16 & 6.85 & 6.07 \\
\hline SRS & $100 \%$ & -57.13 & -46.78 & -32.05 & -56.46 & -55.60 & -25.69 & 12.53 & -37.77 & -11.51 & -18.40 & -31.37 & 13.67 & -82.35 & -63.74 & -53.37 & -67.00 & -27.00 & -33.37 \\
\hline SMN & $80 \%$ & -52.23 & -46.41 & -28.58 & -41.57 & -31.00 & -12.29 & -21.88 & -26.56 & -24.66 & -26.99 & -29.92 & 1.28 & -70.54 & -70.25 & -42.52 & -53.02 & -4.48 & -31.21 \\
\hline SKF & $92 \%$ & -36.44 & -45.52 & -40.15 & -43.44 & -42.26 & 46.52 & 45.30 & -3.16 & -14.97 & -10.18 & -8.15 & 25.69 & -69.42 & -58.59 & -54.83 & -21.71 & -5.07 & 17.57 \\
\hline DUG & $72 \%$ & -44.74 & -35.74 & -29.06 & -36.62 & -21.23 & -32.78 & -27.58 & -7.12 & -2.97 & 7.56 & -7.15 & 7.25 & -56.51 & -50.05 & -42.60 & -50.89 & -14.56 & -44.83 \\
\hline
\end{tabular}

b. Latin America Category

\begin{tabular}{|c|c|c|c|c|c|c|c|c|c|c|c|c|c|c|c|c|c|c|c|}
\hline \multirow{3}{*}{$\begin{array}{l}\text { Ticker } \\
\text { EWZ }\end{array}$} & \multirow{3}{*}{$\begin{array}{l}\text { Volatility } \\
47 \%\end{array}$} & \multicolumn{6}{|c|}{ Overall (19/03/07-17/03/10) } & \multicolumn{6}{|c|}{ Pre-Tsunami (19/03/07-17/09/08) } & \multicolumn{6}{|c|}{ Post- Tsunami(18/09/08-17/03/10) } \\
\hline & & Buy-hold & OBV10 & $0 \mathrm{OBV} 20$ & OBV50 & OBV100 & OBVMA10 $\times 20$ & Buy-hold & OBV10 & OBV20 & OBV50 & OBV1 & 0 OBVMA $10 \times 20$ & Buy-hold & OBV10 & o OBV20 & OBV50 & 30 OBV100 & OVMA10 $\times 20$ \\
\hline & & 16.52 & -5.76 & 9.73 & 17.20 & 27.02 & -1.29 & 9.58 & -1.39 & -2.29 & -8.07 & 5.99 & 6.81 & 21.34 & -3.74 & 23.39 & 49.80 & 52.51 & 23.30 \\
\hline ILF & $51 \%$ & 12.10 & -10.03 & 37.85 & 23.15 & 19.52 & 12.94 & 4.11 & -10.28 & 4.11 & 4.52 & 3.22 & 30.14 & 15.76 & -2.63 & 11.75 & 45.36 & 38.61 & 50.22 \\
\hline
\end{tabular}

c. Telecommunications Category

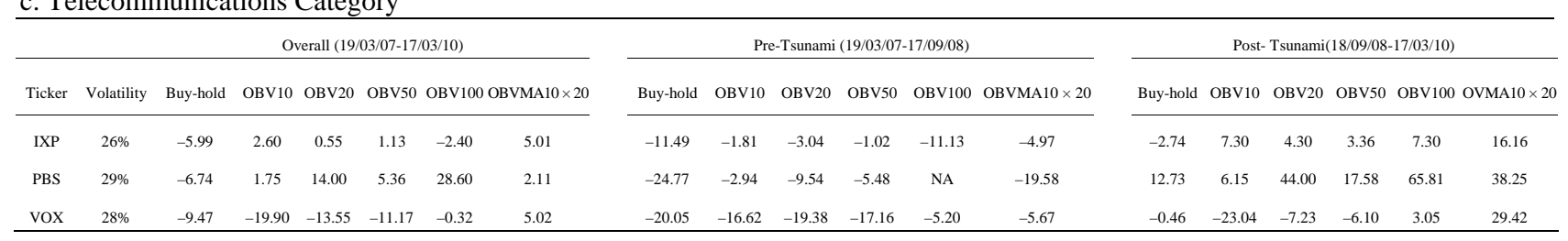

d. Consumer Staples Category

\begin{tabular}{|c|c|c|c|c|c|c|c|c|c|c|c|c|c|c|c|c|c|c|c|}
\hline \multirow[b]{2}{*}{ Ticker } & \multirow[b]{2}{*}{ Volatility } & \multicolumn{6}{|c|}{ Overall (19/03/07-17/03/10) } & \multicolumn{6}{|c|}{ Pre-Tsunami (19/03/07-17/09/08) } & \multicolumn{6}{|c|}{ Post- Tsunami(18/09/08-17/03/10) } \\
\hline & & Buy-hold & OBV10 & OBV20 & OBV50 & 0 OBV100 & OBVMA10 × 20 & Buy-hold & OBV10 & OBV20 & OBV50 & OBV100 & OBVMA10 $\times 20$ & Buy-hold & OBV10 & OBV20 & OBV50 & OBV100 & OVMA $10 \times 20$ \\
\hline VDC & $17 \%$ & 2.62 & -3.77 & -4.03 & -5.70 & 3.51 & 0.09 & 4.06 & -7.21 & -7.59 & -7.12 & 0.67 & 3.55 & -0.11 & -0.17 & -0.30 & 0.04 & 18.28 & -0.11 \\
\hline XLP & $17 \%$ & 2.28 & -6.52 & -9.54 & 0.33 & -6.49 & -0.50 & 4.16 & -12.13 & -10.43 & -0.67 & -6.08 & -0.90 & -0.02 & -0.33 & -6.19 & 2.09 & -0.07 & 1.66 \\
\hline
\end{tabular}

\section{e. Bond Category}

\begin{tabular}{|c|c|c|c|c|c|c|c|c|c|c|c|c|c|c|c|c|c|c|c|}
\hline \multirow[b]{2}{*}{ Ticker } & \multicolumn{7}{|c|}{ Overall (19/03/07-17/03/10) } & \multicolumn{6}{|c|}{ Pre-Tsunami (19/03/07-17/09/08) } & \multicolumn{6}{|c|}{ Post- Tsunami(18/09/08-17/03/10) } \\
\hline & Volatility & Buy-hold & OBV10 & OBV20 & OBV50 & OBV100 & BVMA10 $\times 20$ & Buy-hold & OBV10 & OBV20 & OBV50 & OBV100 & OBVMA10 $\times 20$ & Buy-hold & OBV10 & OBV20 & OBV50 & OBV100 & OVMA10 $\times 20$ \\
\hline HYG & $17 \%$ & -5.45 & -3.96 & -0.42 & 2.40 & 2.22 & 2.09 & -15.70 & -6.40 & -1.98 & -3.91 & -4.38 & -1.43 & 0.45 & 6.36 & 6.00 & 11.53 & 9.17 & 13.74 \\
\hline GBF & $7 \%$ & 1.67 & 2.14 & 0.06 & 2.94 & 1.89 & 0.09 & 0.39 & 2.39 & -1.77 & 2.11 & 1.71 & -1.49 & 3.78 & 2.27 & 1.95 & 0.24 & 0.04 & 2.39 \\
\hline BSV & $5 \%$ & 2.31 & -0.01 & 1.71 & -0.48 & -2.20 & -0.32 & 2.61 & -0.14 & 3.24 & 3.38 & -0.25 & 2.28 & 2.13 & 0.23 & 0.35 & -2.58 & 0.81 & -0.96 \\
\hline BLV & $12 \%$ & 1.35 & -1.95 & -1.14 & -0.51 & -1.92 & -1.18 & -0.38 & -4.71 & -2.73 & -2.90 & -5.21 & -7.68 & 3.08 & 0.85 & 2.29 & 2.58 & 2.96 & 8.79 \\
\hline BIV & $9 \%$ & 2.61 & 2.07 & 3.75 & 3.27 & 1.22 & 4.23 & 1.51 & 0.43 & 2.09 & 3.93 & $\mathrm{NA}$ & 4.36 & 4.75 & 3.69 & 5.39 & 2.62 & 1.24 & 5.90 \\
\hline CSJ & $6 \%$ & 1.19 & 1.32 & -0.50 & 1.34 & 0.30 & 1.47 & -2.09 & 0.12 & -2.70 & -0.71 & -1.67 & -1.98 & 5.38 & 6.13 & 3.88 & 2.52 & 1.90 & 2.95 \\
\hline GVI & $6 \%$ & 1.95 & 0.22 & 2.00 & 0.24 & -0.10 & 2.64 & 1.18 & -1.32 & -0.15 & -0.95 & -0.45 & -0.01 & 3.17 & 1.48 & 3.91 & 0.93 & 1.06 & 6.39 \\
\hline LQD & $11 \%$ & -0.16 & 3.86 & 1.32 & -9.47 & -1.12 & -1.89 & -11.75 & -4.45 & -3.92 & -8.71 & -7.96 & -9.55 & 12.10 & 6.94 & 6.91 & -1.63 & 3.03 & 4.70 \\
\hline TIP & $9 \%$ & 1.24 & -2.39 & -0.31 & 0.57 & -1.67 & 0.12 & 2.61 & 2.30 & -0.82 & 2.07 & -3.33 & -0.89 & 0.76 & -4.40 & 3.10 & -0.92 & 2.15 & 4.58 \\
\hline MBB & $4 \%$ & 2.16 & -2.34 & 0.07 & 0.75 & 2.13 & 0.11 & 1.75 & -2.87 & 1.13 & 1.96 & 1.04 & -0.19 & 3.23 & -0.75 & 0.33 & 0.74 & 2.60 & 2.10 \\
\hline
\end{tabular}

\section{f. US Treasury Bill Category}

\begin{tabular}{|c|c|c|c|c|c|c|c|c|c|c|c|c|c|c|c|c|c|c|c|}
\hline \multirow[b]{2}{*}{ Ticker } & \multicolumn{7}{|c|}{ Overall (19/03/07-17/03/10) } & \multicolumn{6}{|c|}{ Pre-Tsunami (19/03/07-17/09/08) } & \multicolumn{6}{|c|}{ Post- Tsunami(18/09/08-17/03/10) } \\
\hline & Volatility & Buy-hold & OBV10 & OBV20 & OBV50 & OBV100 & OBVMA10 $\times 20$ & Buy-hold & OBV10 & OBV20 & OBV50 & OBV100 & OBVMA $10 \times 20$ & Buy-hold & OBV10 & OBV20 & OBV50 & OBV100 & DVMA10 $\times 20$ \\
\hline IEI & $6 \%$ & 3.48 & 2.62 & 1.62 & 2.05 & 0.91 & 2.21 & 6.24 & 0.76 & 1.53 & 4.56 & 0.08 & 4.88 & 1.16 & 4.53 & -1.95 & -3.08 & 0.77 & 0.09 \\
\hline SHV & $1 \%$ & 0.34 & -0.06 & 0.01 & -0.26 & -0.12 & 0.21 & 0.87 & 0.38 & 0.60 & $\mathrm{NA}$ & NA & 0.67 & -0.17 & -0.42 & -0.44 & -0.25 & -0.19 & -0.21 \\
\hline SHY & $2 \%$ & 1.28 & -0.87 & 0.00 & 0.12 & -0.42 & 0.39 & 3.01 & -0.81 & 1.69 & 1.74 & 0.16 & 2.01 & -0.22 & -0.73 & -1.45 & -1.10 & -0.28 & -0.86 \\
\hline TLH & $11 \%$ & 2.48 & -4.99 & -0.62 & -3.45 & 0.35 & -0.45 & 5.78 & -3.61 & 4.00 & 1.85 & 2.86 & 4.53 & -0.12 & -5.38 & -1.75 & -5.28 & -1.99 & -1.57 \\
\hline TLT & $15 \%$ & 0.66 & -1.24 & -1.55 & 0.33 & -8.04 & -1.70 & 6.37 & 4.12 & 2.69 & 0.99 & -1.56 & 1.76 & -3.86 & -6.38 & -2.66 & -0.36 & -8.97 & -4.87 \\
\hline
\end{tabular}

\section{Results and Conclusions}

Table 2 reports the annualized rate of return (in percent- age) of our $O B V$ trading rules. Note that the $O B V M A 10 \times$ 20 rule performs consistently well in the three periods. For Bear Market and Latin America ETFs, the trading 
rules outstrip the buy-and-hold benchmark in almost all cases. Specifically, for the case of Proshares Ultrashort Financials (SKF), the OBVMA10 $\times 20$ is able to achieve an annual rate of return of $47 \%$. For the Bear Market ETFs, all the trading rules, especially OBVMA10 $\times 20$, clearly dominate the buy-and-hold strategy. For ETFs in Bond, Telecommunications and Consumers Staples, our trading rules also beat the market in general. Note that the OBV trading rules excel in volatile markets. The higher the volatility, the better that the relative performance of the $O B V$ rules. For ETFs associated with US Treasury Bills, the $O B V$ trading rules fail to deliver a handsome return. For the whole sample period and pre-Tsunami period, the profits generated by the $O B V$ trading rules are reasonably good. For the post-Tsunami period, our trading rules generate notably higher return than the benchmark. Overall, our results reveal that the $O B V$ trading rule can beat the buy-and-hold strategy in the ETF market, especially for those ETFs with high volatility. This is in contrast to the conventional wisdom that funds should be bought and held.

\section{References}

[1] L. Carrel, "ETFs for the Long Run: What They Are, How They Work, and Simple Strategies for Successful LongTerm Investing,” Wiley, New York, 2008.

[2] D. Wagner, "Trading ETFs: Gaining an Edge with Technical Analysis (Bloomberg Market Essentials: Technical Analysis),” Bloomberg Press, New York, 2008.

[3] W. W. H. Tsang and T. T. L. Chong, "Profitability of the On-Balance Volume Indicator,” Economics Bulletin, Vol. 29, No. 3, 2009, pp. 2424-2431.

[4] J. H. Poterba and J. B. Shoven, "Exchange-Traded Funds: A New Investment Option for Taxable Investors,” American Economic Review, Vol. 92, No. 2, 2002, pp. 422-427. doi:10.1257/000282802320191732

[5] W. J. Trainor Jr., "Do Leveraged ETFs Increase Volatility,” Technology and Investment, Vol. 1, No. 3, 2010, pp. 215-220. doi:10.4236/ti.2010.13026

[6] W. Brock, J. Lakonishok and B. LeBaron, "Simple Technical Trading Rules and the Stochastic Properties of Stock Returns,” Journal of Finance, Vol. 47, No. 5, 1992, pp. 1731-1764. $\underline{\text { doi:10.2307/2328994 }}$ 